\title{
ORIGINAL RESEARCH \\ Comparison of 2-Year Angiographic Outcomes of Stent- and Nonstent-Assisted Coil Embolization in Unruptured Aneurysms with an Unfavorable Configuration for Coiling
}

\author{
G. Hwang \\ H. Park \\ J.S. Bang \\ S.-C. Jin \\ B.C. Kim \\ C.W. Oh \\ H.S. Kang \\ M.H. Han \\ O.-K. Kwon
}

BACKGROUND AND PURPOSE: Stents are known to have hemodynamic and biologic effects in addition to their mechanical scaffold effect. To determine whether stents affect long-term outcomes after coiling of unruptured aneurysms, we compared angiographic outcomes at 2 years postembolization for stent- and nonstent-assisted coiled unruptured aneurysms.

MATERIALS AND METHODS: Stent-assisted coiling was used in unruptured aneurysms unfavorable for simple coiling (neck size $>4 \mathrm{~mm}$ and dome-to-neck ratio $<1.5$ ) in our practice. Therefore, 126 coiled unruptured aneurysms in total (40 [31.7\%] stent group and 86 [68.3\%] nonstent group) with these conditions were selected for this study. The nonstent group aneurysms were treated with multiple microcatheter technique (53 cases) or balloon-assisted technique (33 cases). Self-expandable stents were used for coiling in stent group aneurysms. No significant difference in aneurysmal characteristics (aneurysm type [sidewall/bifurcation], diameter, neck size, and dome-to-neck ratio) or angiographic outcome at embolization (packing attenuation, obliteration grade, and contrast filling) were observed between the 2 study groups.

RESULTS: At 2-year follow-up visits, rates of progressive occlusion (stent group, 17/40 [42.5\%] versus nonstent group, 34/86 [39.5\%]) and recanalization (7/40 [17.5\%] versus 18/86 [21.0\%]) did not show a statistically significant difference between the 2 groups $(P=.895)$.

CoNCLUSIONS: The present study did not show that additional hemodynamic and biologic effects of stents designed for neck remodeling were enough to enhance progressive occlusion and prevent the recanalization of unruptured aneurysms. Our finding suggests that stent placement provides no better long-term angiographic outcomes for unruptured aneurysms with an unfavorable configuration for coiling.

ABBREVIATIONS: DSA $=$ digital subtraction angiography; IU $=$ international unit; $\mathrm{MRA}=\mathrm{MR}$ angiography

D uring the embolization of saccular aneurysms, stents are used for neck remodeling and serve as a scaffold for the coils. In addition to neck remodeling, stents are known to have additional hemodynamic ${ }^{1-6}$ and biologic effects. ${ }^{6-8}$ However, little data are available to demonstrate whether these effects influence the long-term angiographic outcomes of coiled aneurysms, especially of unruptured aneurysms unfavorable for simple coiling (wide neck [ $>4 \mathrm{~mm}$ ] with a dome-to-neck ratio of $<1.5$ ), in which a stent is generally used. In our practice, we have treated such aneurysms with various endovascular methods, including the multiple microcatheter technique and balloon- and stent-assisted emboliza-

Received December 24, 2010; accepted after revision January 31, 2011

From the Department of Neurosurgery (G.H.), Chuncheon Sacred Heart Hospital, Hallym University and Graduate School of Gangwon University, Chuncheon, Korea; Department of Neurosurgery (H.P., J.S.B., C.W.O., O.-K.K.), Seoul National University Bundang Hospital, Seongnam, Korea; Department of Neurosurgery (S.-C.J.), Haeundae Paik Hospital, Inje University, Busan, Korea; Department of Neurosurgery (B.C.K.), Dongguk University IIsan Hospital, Goyang, Korea; and Department of Neurosurgery (H.S.K.) and Radiology (M.H.H.), Seoul National University Hospital, Seoul, Korea.

This study was supported by the Korea Health 21 R\&D Project, Ministry of Health, Welfare and Family Affairs, Republic of Korea (grant A06-0171-B51004-06N1-00040B).

Please address correspondence to O-Ki Kwon, MD, PhD, Department of Neurosurgery, Seoul National University Bundang Hospital, 300 Gumi-dong, Bungdang-gu, Seongnam, Gyeonggi, 463-707, Korea; e-mail: kwonoki@snu.ac.kr

Indicates open access to non-subscribers at www.ajnr.org

http://dx.doi.org/10.3174/ajnr.A2592 tion. In this article, we compared the 2-year follow-up angiographic outcomes between stent- and nonstent-assisted coil embolization of unruptured aneurysms with an unfavorable configuration.

\section{Materials and Methods}

\section{Patient Selection}

Our institutional review board approved this study. We retrospectively reviewed the clinical and radiologic data of patients with unruptured aneurysms who underwent endosaccular coil embolization between May 2003 and January 2008 at our institute. Because the thrombogenic environment could have affected initial angiographic results, unruptured aneurysms coiled at the same time as a ruptured aneurysm was treated were excluded. In addition, patients who did not undergo a conventional angiographic evaluation at 2 years after coil embolization also were excluded.

In our practice, stent-assisted coiling was used in unruptured aneurysms under the following conditions: $>4 \mathrm{~mm}$ in neck size and a dometo-neck ratio of $<1.5$. However, many aneurysms with these conditions also were coiled without stent assistance, when the aneurysm was expected to be able to contain the coil mass. In cases with a small neck $(\leq 4 \mathrm{~mm})$, surgical obliteration was used if the aneurysm was unfavorable for simple coiling and could be coiled under only stent assistance. For comparison therefore, we included unruptured aneurysms treated by nonstent-assisted coiling, which had the same conditions as those treated by stent-assisted coiling, in this study. Eventually, 126 aneurysms in total in 121 patients were included in this study (Fig 1). 


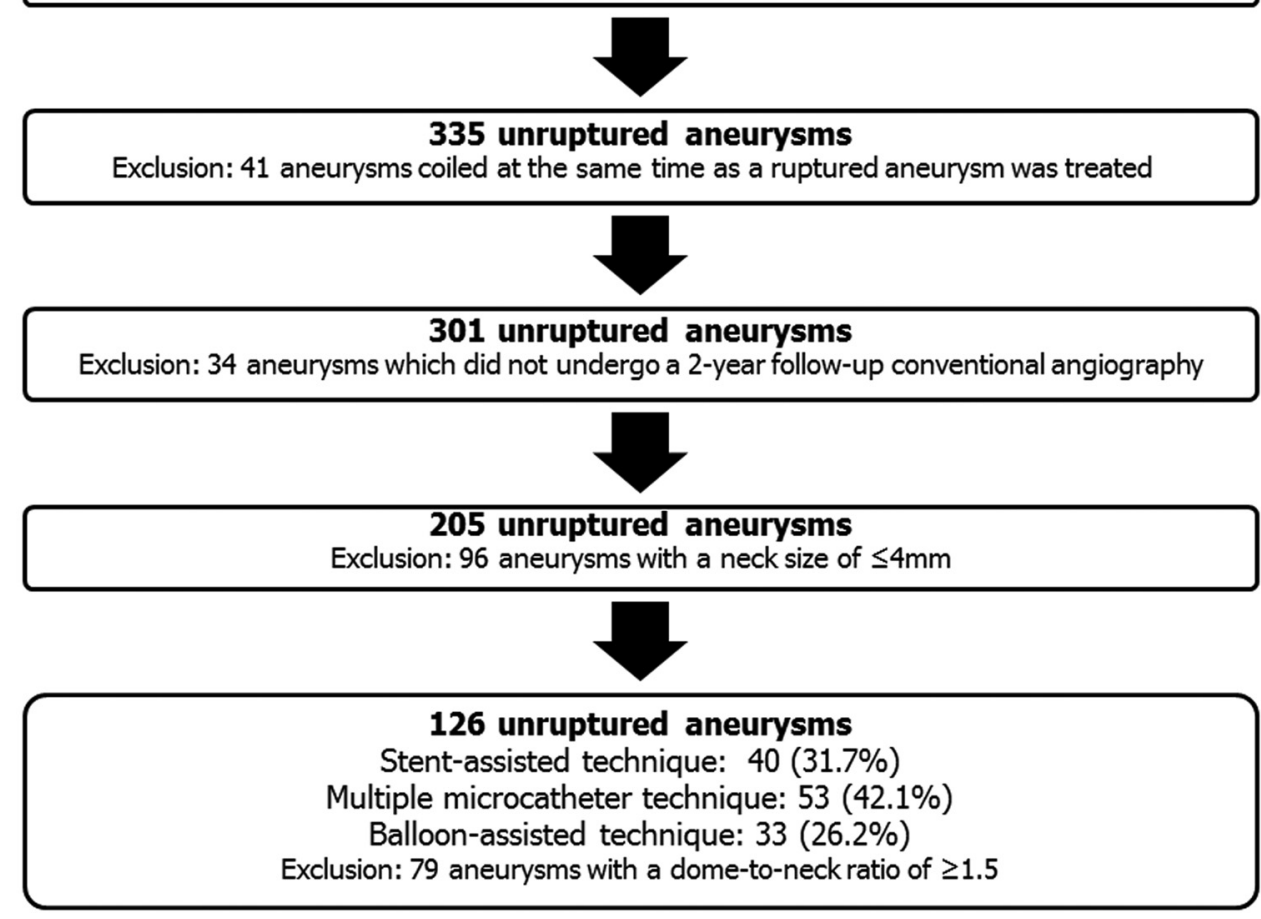

Fig 1. Patient flow according to exclusion criteria for this study.

\section{Coil Embolization Procedure}

All aneurysm coilings were performed under general anesthesia by using a biplane angiographic unit (Integris Allura; Philips Healthcare, Best, the Netherlands). Stent-assisted coil embolization was used in $40(31.7 \%)$ aneurysms. In the remaining $86(68.3 \%)$ aneurysms, a multiple microcatheter technique $(53,42.1 \%)$ or balloon-assisted coil embolization technique $(33,26.2 \%)$ was used. Technical details of aneurysm coiling and stent deployment were conventional and have been described in detail previously ${ }^{9,10}$ All aneurysm embolizations were performed by using detachable platinum coils, which included the GDC (Boston Scientific, Fremont, California), MicroPlex (MicroVention, Aliso Viejo, California), Trufill-DCS (Cordis, Miami Lakes, Florida), and Axium (ev3, Irvine, California) coils. Modified coils, such as Matrix (Boston Scientific) and HydroCoil (MicroVention), were not used. The stents used for remodeling were the Neuroform (Boston Scientific; 17 cases, $42.5 \%$ ) or Enterprise (Cordis; 23 cases, 57.5\%) and were successfully deployed to cover the aneurysmal neck in all cases. In no case was a stent overlapping used. In every case, systemic heparinization was performed after placing a femoral introducer sheath. In accord with our embolization protocol, 3000 IUs of heparin was administered as an intravenous bolus injection, and this was followed by an additional 1000 IUs per hour. Heparin was discontinued after embolization in all patients. When stent-assisted coil embolization was planned, we prescribed antiplatelet medication (oral aspirin, $100 \mathrm{mg}$ and clopidogrel, $75 \mathrm{mg}$ ) daily for at least 5 days before coiling. If these daily regimens were not followed, aspirin and clopidogrel were administered at loading doses of 300-500 and $300 \mathrm{mg}$, respectively. For daily antiplatelet medication after stent deployment, 2 drugs (aspirin, $100 \mathrm{mg}$ and clopidogrel, $75 \mathrm{mg}$ ) were used for the first 3 months, and then aspirin at $100 \mathrm{mg}$ was continued.

\begin{tabular}{|c|c|c|}
\hline & $\begin{array}{c}\text { Stent- } \\
\text { Assisted } \\
(\%), n=40\end{array}$ & $\begin{array}{l}\text { Nonstent- } \\
\text { Assisted } \\
(\%), n=86\end{array}$ \\
\hline Internal carotid artery & $25(62.5)$ & $50(62.8)$ \\
\hline Cavernous & $5(12.5)$ & $9(11.6)$ \\
\hline Proximal intradural or ophthalmic & $16(40.0)$ & $30(37.2)$ \\
\hline Posterior communicating & $2(5.0)$ & $6(7.0)$ \\
\hline Anterior choroidal & & $1(2.3)$ \\
\hline Bifurcation & $2(5.0)$ & $4(4.7)$ \\
\hline Middle cerebral artery & $2(5.0)$ & $6(8.1)$ \\
\hline Bifurcation & $2(5.0)$ & $6(8.1)$ \\
\hline Anterior cerebral artery & $2(5.0)$ & $6(8.1)$ \\
\hline Anterior communicating & $2(5.0)$ & $6(8.1)$ \\
\hline Posterior circulation & $11(27.5)$ & $17(21.0)$ \\
\hline Basilar bifurcation & $7(17.5)$ & $11(14.0)$ \\
\hline Others & $4(10.0)$ & $6(7.0)$ \\
\hline
\end{tabular}

\section{Aneurysmal Characteristics and Initial Angiographic Outcomes}

The following aneurysmal characteristics were recorded; aneurysm location (Table 1), aneurysm type (sidewall, bifurcation), diameter, neck size, dome-to-neck ratio, and volume. Aneurysm obliteration grade ( 0 , complete; 1 , occlusion $\geq 90 \%$; 2 , occlusion $70 \%-89 \%$; 3 , occlusion $50 \%-69 \%$; 4 , occlusion $25 \%-49 \%$; and 5 , occlusion $<25 \%$ ), contrast filling within the coil mass, and packing attenuation (percentage) were checked to evaluate initial angiographic outcome. ${ }^{11}$

The following formulas were used to calculate aneurysm volumes and packing densities.

Aneurysmal volume $(c c)=4 / 3 \times \pi \times[$ length $] / 2 / 10$

$\times[$ width $] / 2 / 10 \times[$ height $] / 2 / 10$ 
Coil volume $(c c)=\sum \pi \times([$ coil outer diameter $] / 2 \times$

$$
2.54)^{2} \times[\text { coil length }]
$$

Packing attenuation $(\%)=$

$$
\text { [coil volume]/[aneurysm volume }] \times 100
$$

\section{Imaging Follow-Up after Coil Embolization}

The imaging tools used for follow-up were skull imaging by using an angiographic unit (working, conventional frontal, and lateral projections), MRA, and DSA. Skull radiographs were taken at 3, 9, 15, and 21 months and MRA at 6,12 , and 18 months postembolization. DSA was performed at 2 years postembolization. Whenever findings were obtained that suggested recanalization on skull images or MRA images, DSA also was performed.

Follow-up DSA images were obtained in the previous working projection and in conventional frontal and lateral projections. Rotational angiography was performed to detect any change in angiographic outcome. DSA also was performed at the projections determined optimal by rotational angiography.

\section{Evaluation of Angiographic Outcomes at 2 Years Postembolization}

Changes in angiographic outcome were evaluated by DSA and were classified as follows: stable (no change in coil configuration, obliteration grade, or contrast filling), improved (progressive occlusion or involution of the neck remnant or contrast filling in aneurysm), and recanalized (aneurysm recurrence evident due to neck growth, coil compaction, coil extrusion by aneurysm degradation, ${ }^{12}$ or new sac formation). ${ }^{11}$ In addition, newly visualized or increased contrast filling inside an aneurysm also was considered to indicate recanalization. All radiologic data were reviewed by a neuroradiologist (M.H.H.), who was unaware of clinical results.

\section{Statistical Analyses}

Statistical analysis was conducted by using SPSS version 17 (SPSS, Chicago, Illinois). The Mann-Whitney $U$ test was used for continuous variables, and the $\chi^{2}$ or Fisher exact test were used for nominal factors. Statistical significance was accepted for $P$ values of $<0.05$.

\section{Results}

Basic demographic data, aneurysmal characteristics, and initial angiographic outcomes after embolization are summarized in Table 2. No significant difference was observed between the stentand nonstent-assisted coil embolization groups.

Over 2 years after coil embolization, angiographic outcomes had changed in both groups (Table 3). Rates of angiographic improvement due to progressive occlusion (17/40 [42.5\%] versus $34 / 86[39.5 \%])$ and recanalization (7/40 [17.5\%] versus 18/86 [21.0\%]) were slightly more favorable in the stent-assisted group than in the nonstent-assisted group. However, these changes were not found significantly different $(P=.895)$.

Of 51 coiled unruptured aneurysms showing angiographic improvement, 46 (90.2\%) were sidewall-type unruptured aneurysms. This improvement occurred even in nonstent-assisted coil embolized aneurysms and its rate was similar with that in stentassisted coil embolized aneurysms $(P=1.000$; Table 3$)$. In contrast, recanalization frequently developed in bifurcation-type aneurysms $(17 / 25,68 \%)$. This recanalization rate in coiled unruptured aneurysms protected by stent was not lower than that in the nonstent-assisted group $(P=1.000)$.
Table 2: Basic demographic data, aneurysmal characteristics, and initial angiographic outcomes for the stent- and nonstent-assisted coil embolization groups

\begin{tabular}{lccr}
\hline & $\begin{array}{c}\text { Stent- } \\
\text { Assisted } \\
(\%), n=40\end{array}$ & $\begin{array}{c}\text { Nonstent- } \\
\text { Assisted } \\
(\%), n=86)\end{array}$ & $\begin{array}{c}P \\
\text { Value }\end{array}$ \\
\hline Male & $10(25.0)$ & $23(26.7)$ & 1.000 \\
Age & $56.5 \pm 10.28$ & $59.4 \pm 9.83$ & .126 \\
Aneurysm type & & & .316 \\
$\quad$ Sidewall & $29(72.5)$ & $53(61.6)$ & \\
$\quad$ Bifurcation & $11(27.5)$ & $33(38.4)$ & .687 \\
Diameter (mm) & $7.7 \pm 3.06$ & $7.5 \pm 2.53$ & .180 \\
Neck size (mm) & $6.1 \pm 2.26$ & $5.6 \pm 1.82$ & .178 \\
Dome-to-neck ratio & $0.89 \pm 0.283$ & $0.95 \pm 0.211$ & .160 \\
Packing density (\%) & $29.1 \pm 14.59$ & $27.8 \pm 9.50$ & \\
Obliteration grade & & & \\
0 & $6(15.0)$ & $6(7.0)$ & .566 \\
1 & $14(35.0)$ & $27(31.4)$ & \\
2 & $14(35.0)$ & $46(53.5)$ & $7(8.1)$ \\
3-5 & $6(15.0)$ & $41(47.7)$ & \\
Contrast filling & $22(55.5)$ & & \\
\hline
\end{tabular}

Table 3: Changes in angiographic outcomes in the stent- and nonstent-assisted coil embolization groups at 2 years postcoil embolization

\begin{tabular}{llccr}
\hline & & $\begin{array}{c}\text { Stent- } \\
\text { Assisted } \\
(\%)\end{array}$ & $\begin{array}{c}\text { Nonstent- } \\
\text { Assisted } \\
(\%)\end{array}$ & $\begin{array}{c}P \\
\text { Value }\end{array}$ \\
\hline Total $(n=126)$ & Stable & $16 / 40(40.0)$ & $34 / 86(39.5)$ & $.895^{\mathrm{a}}$ \\
& Improved & $17 / 40(42.5)$ & $34 / 86(39.5)$ & \\
Sidewall $(n=82)$ & Recanalized & $7 / 40(17.5)$ & $18 / 86(21.0)$ & \\
& Improved & $16 / 29(55.2)$ & $30 / 53(55.6)$ & $1.000^{\mathrm{b}}$ \\
Bifurcation $(n=44)$ & Recanalized & $3 / 29(10.3)$ & $5 / 53(9.4)$ & $1.000^{\mathrm{b}}$ \\
& Improved & $1 / 11(9.1)$ & $4 / 33(12.1)$ & $1.000^{\mathrm{b}}$ \\
& Recanalized & $4 / 11(36.4)$ & $13 / 33(39.4)$ & $1.000^{\mathrm{b}}$ \\
\hline
\end{tabular}

Note:-Stable $=$ no change in coil configuration, obliteration grade, or contrast filling improved = progressive occlusion or involution in the neck remnant or contrast filling in aneurysm; recanalized = aneurysm recurrence evident by neck growth, coil compaction, coil degradation, or new sac formation.

${ }^{2}$ test.

a $\chi^{2}$ test.
Fisher exact test.

Retreatment was performed in 7 of 25 aneurysms that showed recanalization (28\%; stent-assisted, $2 / 7$ [28.6\%] versus nonstent-assisted, 5/18 [27.8\%], $P=1.000)$. No significant long-term angiographic outcome difference was observed between stent types (Neuroform versus Enterprise) (stable, $7 / 17$ [41.2\%] versus 9/23 [39.1\%]; improved, $7 / 17$ [41.2\%] versus 10/23 [43.5\%]; recanalized, $3 / 17$ [17.6\%] versus $4 / 23[17.4 \%] ; P=.989)$.

In the initial coil embolization, thromboembolic complication occurred in $5(4.0 \%)$ cases (stent-assisted group, 3 [7.5\%] and nonstent-assisted group, 2 [2.3\%]). There was no hemorrhagic complication including aneurysm rupture during the procedures. Over 2 years after coiling, no in-stent stenosis or branch vessel occlusion developed in the stentassisted group and coil loop migration into parent artery without flow disturbance was detected in $1(1.2 \%)$ of the nonstent-assisted group. No aneurysm bled in either group.

\section{Discussion}

Aneurysms unfavorable for simple coiling can be embolized by various methods, besides stent-assisted coiling. However, these methods are relatively complex with long procedure times. Contrarily, once a stent is deployed across the aneurys- 
mal neck, coils can be placed within the aneurysmal sac more easily with less concern of coil migration. As well as this mechanical effect, experimental studies have demonstrated that stents have hemodynamic ${ }^{1-6}$ and biologic effects. ${ }^{6-8}$ In theory, flow diversion can cause blood to become stagnant and lead to progressive thrombosis and the promotion of endothelialization that can help the aneurysmal neck close. Accordingly, these additional effects of stents may enhance the durability of coiled aneurysms. Clinically, however, it is unclear whether these effects really improve long-term outcome, especially when one considers that the stents designed for neck modeling have large porosities. A previous observational study reported favorable progressive thrombosis and recanalization rates for aneurysms over a relatively short follow-up (average, 4.6 months) after stent-assisted embolization, ${ }^{13}$ but progressive occlusion by thrombosis also occurs even in aneurysms treated with coils only. ${ }^{11,14}$ Thus, we conducted this comparative study to determine the actual effect of a stent. In this study, we focused on unruptured aneurysms unfavorable for coiling, because this aneurysm type is generally treated by stent-assisted coil embolization.

In the present study, the following findings were notable. First, in unruptured aneurysms with an unfavorable configuration for coiling ( $>4 \mathrm{~mm}$ in neck size and a dome-to-neck ratio of $<1.5$ ), no statistically significant difference was shown in 2-year angiographic outcome between stent- and nonstentassisted coil embolization. Recanalization rates in both groups were similar to long-term outcome in previous historical series. ${ }^{15}$ Second, angiographic improvement due to progressive thrombosis, which was frequently observed in sidewall-type aneurysms, was not unusual even in coiled unruptured aneurysms not covered with stent. Finally, recanalization was frequent even in coiled unruptured aneurysms protected by stent, especially bifurcation-type aneurysms. These results suggest that the hemodynamic and biologic effects of stents used during coil embolization are not enough to expect additional improvement of occlusion status and prevention of the recanalization in real patients. Therefore, even when a stent is used, compact coil packing should be used to treat unruptured saccular aneurysms to achieve best long-term outcomes.

The present study has several limitations. First, it is limited by its retrospective nature and a relatively small cohort. Second, there is a previous study that suggested that stents lead to a better long-term angiographic outcome in small aneurysms. ${ }^{16}$ In this study, we only included aneurysms $>4 \mathrm{~mm}$ in neck size, because small unruptured aneurysms were not frequently indicated for stent-assisted coiling in our practice. Therefore, this study only represents the unruptured aneurysms with $\geq 4 \mathrm{~mm}$ in neck size and a dome-to-neck ratio of $<1.5$. Last, the morphology of aneurysms treated by stentassisted coiling was not absolutely the same as that of aneurysms treated by multiple microcatheter technique or balloonassisted coiling. Therefore, there may be hidden factors or conditions that we did not consider, though we included many known factors that may influence long-term angiographic outcomes. Consequently, nonanalyzed differences between the groups may affect results. Despite these study limitations, we think that our study is sufficient to provide the necessity for a large randomized control study. There is a report showing different results, in which stents were associated with a signif- icant decrease of angiographic recurrence (stent-assisted group, $14.9 \%$ versus nonstent-assisted group, 33.5\%). ${ }^{17}$ However, it should be considered that their recanalization rate of nonstent assisted coil embolization was higher than that in the present study $(21.0 \%)$ and another study $(24.3 \%)^{16}$ that found no statistically significant difference in recanalization rates between 2 groups. In addition, it is also of note that mean follow-up duration of the stent-assisted group (12 months; range, 1-77 months) was far shorter than that of nonstentassisted group (22 months; range, 1-77 months). Therefore, the long-term effect of stents should be confirmed by a large randomized control study.

\section{Conclusions}

Stents commonly used for neck remodeling facilitate stable coiling and compact packing in unfavorable aneurysms. Although stents also have hemodynamic and biologic effects, we did not find that these effects were enough to enhance progressive occlusion and reduce the recanalization rate of unruptured aneurysms with an unfavorable configuration for coiling. Our study suggests that stent placement in coil embolization does not significantly improve the long-term angiographic outcome of coiled unruptured aneurysms unfavorable for simple coiling.

Disclosures: Moon Hee Han, Consultant: MicroVention Inc.

\section{References}

1. Canton G, Levy DI, Lasheras JC. Hemodynamic changes due to stent placement in bifurcating intracranial aneurysms. J Neurosurg 2005;103:146-55

2. Canton G, Levy DI, Lasheras JC, et al. Flow changes caused by the sequential placement of stents across the neck of sidewall cerebral aneurysms. J Neurosurg 2005; 103:891-902

3. Kim M, Levy EI, Meng H, et al. Quantification of hemodynamic changes induced by virtual placement of multiple stents across a wide-necked basilar trunk aneurysm. Neurosurgery 2007;61:1305-12; discussion 1312-03

4. Lieber BB, Gounis MJ. The physics of endoluminal stenting in the treatment of cerebrovascular aneurysms. Neurol Res 2002;24 Suppl 1:S33-42

5. Lieber BB, Livescu V, Hopkins LN, et al. Particle image velocimetry assessment of stent design influence on intra-aneurysmal flow. Ann Biomed Eng 2002;30:768-77

6. Wanke I, Forsting M. Stents for intracranial wide-necked aneurysms: more than mechanical protection. Neuroradiology 2008;50:991-98

7. Darsaut T, Salazkin I, Ogoudikpe C, et al. Effects of stenting the parent artery on aneurysm filling and gene expression of various potential factors involved in healing of experimental aneurysms. Interv Neuroradiol 2006;12:289-302

8. Lopes D, Sani S. Histological postmortem study of an internal carotid artery aneurysm treated with the Neuroform stent. Neurosurgery 2005;56:E416; discussion E416

9. Im SH, Han MH, Kwon OK, et al. Endovascular coil embolization of 435 small asymptomatic unruptured intracranial aneurysms: procedural morbidity and patient outcome. AJNR Am J Neuroradiol 2009;30:79-84

10. Kwon OK, Kim SH, Kwon BJ, et al. Endovascular treatment of wide-necked aneurysms by using two microcatheters: techniques and outcomes in 25 patients. AJNR Am J Neuroradiol 2005;26:894-900

11. Meyers PM, Schumacher HC, Higashida RT, et al. Reporting standards for endovascular repair of saccular intracranial cerebral aneurysms. AJNR Am J Neuroradiol 2010;31:E12-24

12. Waldron JS, Halbach VV, Lawton MT. Microsurgical management of incompletely coiled and recurrent aneurysms: trends, techniques, and observations on coil extrusion. Neurosurgery 2009;64:301-15; discussion 315-307

13. Fiorella D, Albuquerque FC, Deshmukh VR, et al. Usefulness of the Neuroform stent for the treatment of cerebral aneurysms: results at initial (3-6-mo) follow-up. Neurosurgery 2005;56:1191-201; discussion 1201-02

14. Raymond J, Guilbert F, Weill A, et al. Follow-up of treated aneurysms: the challenge of recurrences and potential solutions. Neuroimaging Clin N Am 2006;16:513-23, ix

15. Murayama Y, Nien YL, Duckwiler G, et al. Guglielmi detachable coil embolization of cerebral aneurysms: 11 years' experience. J Neurosurg 2003;98:959-66

16. Kim DJ, Suh SH, Lee JW, et al. Influences of stents on the outcome of coil embolized intracranial aneurysms: comparison between a stent-remodeled and non-remodeled treatment. Acta Neurochir (Wien) 2010;152:423-29

17. Piotin M, Blanc R, Spelle L, et al. Stent-assisted coiling of intracranial aneurysms: clinical and angiographic results in 216 consecutive aneurysms. Stroke 2010;41:110-15 